\title{
A new health care professional-based model for medication adherence
}

This article was published in the following Dove Press journal:

Patient Preference and Adherence

\section{Muhammad Amir ${ }^{1,2}$ \\ Zeeshan Feroz ${ }^{2,3}$ \\ Anwar Ejaz Beg ${ }^{2}$}

'Pharmacy Services, Aga Khan

University Hospital, Karachi, Pakistan;

${ }^{2}$ Faculty of Pharmacy, Ziauddin

University, Karachi, Pakistan;

${ }^{3}$ Department of Basic Sciences, King

Saud Bin Abdulaziz University, Riyadh,

Saudi Arabia
Correspondence: Muhammad Amir Pharmacy Services, Aga Khan University Hospital, Stadium Road, Karachi, Sindh 74800, Pakistan

Tel +923343308347

Email mohd_amir80@hotmail.com

\begin{abstract}
This paper presents a practice-based conceptual (theoretical) model to address medication adherence. The study reviews literature for different rationales of medication adherence and its associated factors. It also reviews the interventions involved in improving medication adherence and its effect. It is known that many interventions applied have not received great success owing to the fact that they have not been employed in regular clinical practice. Furthermore, models or theories utilized for different interventions were based on patient perspective only. The model presented in the paper is based on health care professionals' perspective, with an aim to be employed in clinical practice. The model framework is based on five premises on the basis of which the two dynamics, patient and health care professional, work. The model is presented with a graphical representation and exemplary procedural framework. It is also compared to other related procedural models. It is suggested that using such a model will allow medication adherence as an integral part of health care outcomes.
\end{abstract}

Keywords: medication adherence, practice model, health care professional, theoretical concept, pharmacy practice

\section{Introduction}

The patient's own beliefs, cultural values, and personal habits have always been an unprecedented factor in their treatment with medication, and hence their health outcome. Rarely would be physicians be able to recognize a patient's peculiar behavior relating medication administration. Some suspicious physicians depended on laboratory and clinical tests in cases where they felt that patients were not taking medication as instructed and also to overcome issues related to medication administration. Nonetheless, these tests are not clinically or economically applicable all the time., ${ }^{1,2}$

Different terms such as compliance, adherence, persistence, and concordance are used to describe such a behavior of patients. New research was initiated to assist physicians to understand this patient behavior, and interventions were developed to improve it. ${ }^{3}$ Patient-related factors including financial issues, complex prescription, poor understanding or motivation, adverse cultural beliefs, poor health literacy, and forgetfulness were identified and used in patient-oriented medical practice. ${ }^{4}$ Interventions such as motivation (counseling), patient education (lectures, leaflets), reminder techniques (SMS, alarms, emails) via telephonic conversation, electronically, and face-to-face methods were tested to improve adherence. ${ }^{5}$

However, the following issues were found with most of the studies related to this issue: 1. Most studies were patient focused ie, to bring changes in patient perspective, and so rarely were any interventions developed to bring changes from the view of the health care professionals. ${ }^{6,7}$ 
2. Most interventions were found efficacious in one or two disease, but did not prove to be effective in clinical practice and showed nonuniform consistency and an increase in cost and time. ${ }^{8,9}$

3. Interventions were an added process in the health care system process flow, and thus resulted increase in increased time taken by the patient, physician, or pharmacist or an additional health professional, thus adding to the process flow. ${ }^{10}$

4. Short-term mechanisms for follow-up or feedback were used. Medicine administration is a continuous cyclic process in which a patient undergoes periods of adherence and nonadherence during their life.

For our paper, we describe these terms reflecting patient's behavior for medication administration from the perspective of health professionals (Box 1). If only prescribers' attributes (knowledge, understanding, ideology) play roles during prescribing, then it is said to be compliance; in such cases, patients have to be strictly compliant to the instructions of the prescriber. Medication adherence differs from medication compliance in that during the prescription process the prescriber considers patients' specific factors (belief, socioeconomic status, etc.) along with his/her own attributes. However, physician and patient factors play a mutual role in concordance. ${ }^{11}$ Concordance emphasizes on creating treatment goals that have been mutually decided upon by both the physician and the patient. ${ }^{12}$ It was suggested that proposing a different concept that could would involve health professionals more than patients for medication adherence, compliance, and concordance could be applicable in practice in different health care system of different countries (Box 1).

\section{Methodology}

We intended to develop a model or conceptual framework defined as a document that "explains, either graphically or in narrative form, the main things to be studied and the key factors, concepts, or variables; and the presumed relationships among them." 13 The process used for developing it was described by Jabareen ${ }^{14}$ for the conceptual framework analysis.

\section{Box I Compliance, adherence, and concordance}

Compliance: physician decides on his/her own accord

Adherence: physician decides on his/her own accord but considers patient's specific factors

Concordance: prescribing based on goals decided mutually by the physician and the patient
The first aim of the study was accomplished in five steps. In the first step, an extensive literature search was carried out to identify the types of models that were used for developing text-based interventions and their impact for reducing medication nonadherence. The second step involved reviewing all the resulting articles to find the deficiencies in the model and assess their impact on medication nonadherence. Based on these reviews, a survey was conducted to assess the information resulting for the data in the third step. In the fourth step, a model was developed based on the findings of the review and survey. In the last stage, the second aim was accomplished and the developed model was discussed with different health care professionals.

A literature review was conducted as an established standard. ${ }^{15}$ The objective of the literature search was to identify available models and types of interventions employed. The search engines used to identify these models included the following: Google scholar, Cinahl, advanced Google Scholar, the Cochrane Library, PubMed, and Medline. The following terms were used: medication, non-adherence, adherence, compliance, non-compliance, concordance, interventions, model, theory, and model. All relevant articles, such as qualitative and quantitative research, reviews, reports, and opinions, were included in the literature. Since very limited research has been conducted on such focused groups, the literature review was conducted from 1980 to 2017 to include all possible data. Any new research released after 2017 was added with time. All resulting articles were appraised as per the guidelines suggested by the Center of Evidence-Based Medicine.

Based on the reviews, it was observed that most studies were patient oriented. Perspectives of health care professionals were unknown. Hence, a cross-sectional descriptive survey was carried out to determine the perspectives of health care professionals and patients with respect to medication nonadherence. This study was conducted in a community health care center located in Sikandarabad. Patients visiting this facility were generally poor and illiterate. This study was conducted on health care professionals, such as physicians, pharmacists, and nurses, and adult patients who were able to communicate in Urdu. The aim of the study was to determine the three fundamentals of medication nonadherence ie, factors associated with nonadherence, extent of drug information patients would prefer, and medication education. ${ }^{16}$

Based on the literature review and survey-based study, a model was formulated and a document was prepared. This document was reviewed by different health care 
professionals, including physicians, pharmacists, professors, and psychologists.

\section{Conceptual framework and its graphical representation}

\section{Premise of the conceptual framework}

The conceptual framework was based on five premises, which are as follows:

1. Medication adherence is the basis of any pharmacological treatment for all diseases. It is a dynamic process which can alter at any stage of treatment and life.

2. Medication adherence is an indirect factor of clinical outcome. Enhancement in medication adherence will not directly result in expected clinical outcome (which varies with respect to medications, disease severity, and patient genetics).

3. Medication adherence is a multidimensional behavioral disorder which should be assessed, treated, and monitored like any other disorder. If diagnosed, it should be referred to as a disorder which exists comorbid with another disease for which pharmacological medication is required.

4. Medication adherence disorder is the responsibility of a team of health care professionals and patients equally.

5. Medication adherence disorder can be acute or chronic, and so may require different ways of treatment, depending on patient and health professional preferences and resources.
Conceptual framework and its graphical representation of the practice model of concordance The practice model of concordance allows health professionals and patients to be placed in parallel in terms of information, responsibility, and accountability. This practice model is presented as a graphical model (Figure 1), the basis of which can be used to develop a procedural model in a respective health care system. The model is based on a closed environment in a health care system (represented by the outer circle) which is based on two dynamics: health care professional related and patient related. The health care professional dynamic comprises of two participants, while the patient dynamic has one participant. All information remains within these three active participants. The three participants involved are the initiator, mediator, and recipient, with the initiator and provider belonging to the class of health professionals.

\section{Initiator}

The first encounter a patient comes across in this model is with the "initiator," which can be an individual or a team of qualified health care professionals. The initiator will be responsible for assessing the patient for medication adherence, medication regimen, documentation, and regular review of patient's adherence status with the mutual agreement with the patient. The initiator takes the responsibility of creating the environment of concordance and would be responsible for determining which patient has a medication adherence

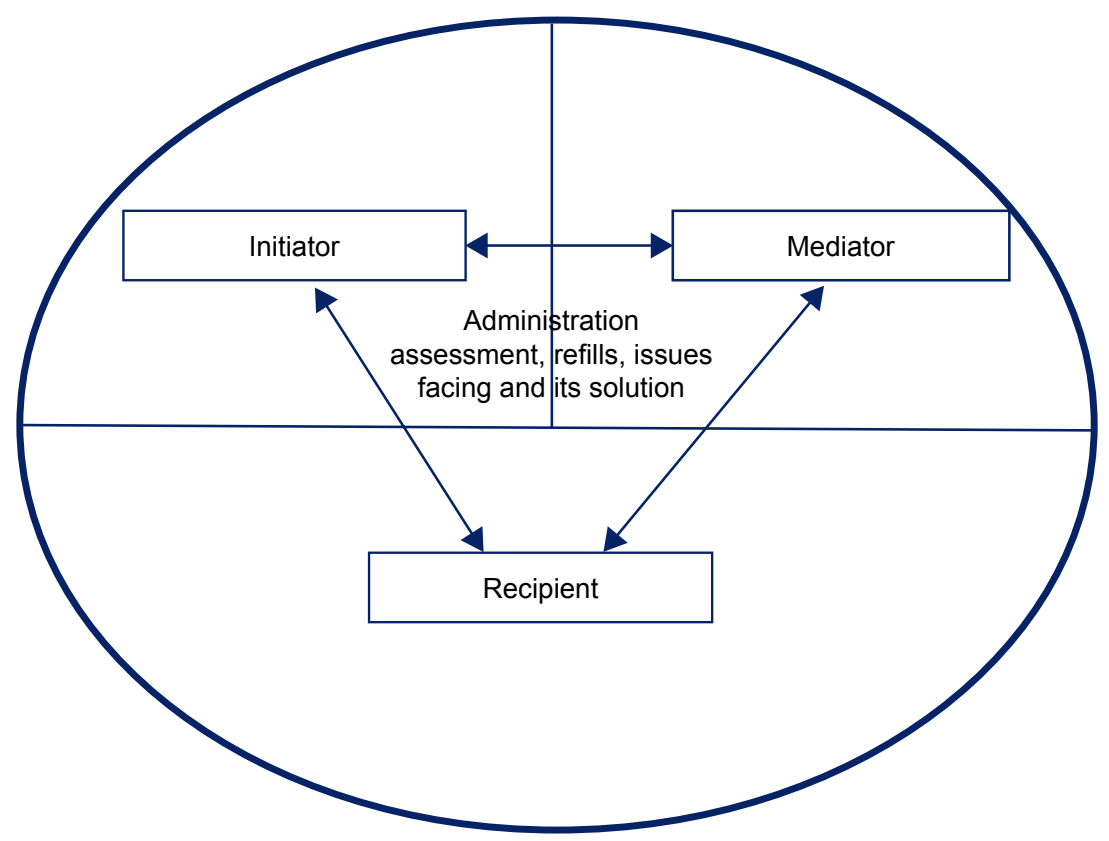

Figure I Graphical representation of practice-based model. 
disorder. The initiator is a part of the process flow of the health care system and should involve a prescriber in it. In different health care systems of different countries, the authority to prescribe medication has been diversified to nurses, pharmacists, and other allied health care professionals. ${ }^{17}$ Thus, this model is not dependent on a physician but on the health professional who prescribes the medication and takes responsibility for its assessment and outcomes.

\section{Mediator}

A mediator is a health professional who is responsible for intervening in case of medication adherence disorder in a patient. The mediator act as the major player who understands the rationale of the disorder and then uses different interventions. The mediator shares the responsibility of monitoring the patient along with the initiator. The mediator should have a close link to medicine supply; however, it does not mean that he/she has to be a pharmacist only. As in different health care settings, nurses or even physicians may be involved in medication supply. The mediator shares equal responsibility with the initiator to achieve the outcome set in the goals.

Moreover, a mediator can be of the same profession as the initiator, but the initiator cannot be a mediator. This is based on the concept of additives ie, one plus one plus is greater than two. The concept of having an initiator and a mediator rather than having a specialized person is also an option. It is thought that having a specialized person for medication adherence will be disadvantageous in term of time and money consumed on additional health professionals. Unlike the initiator where a prescriber should also be involved, a mediator can be from any health care professionals (pharmacist, nurse, physician, psychologist, etc.).

\section{Recipient}

The recipient usually is the one who has been identified as having medication adherence issue and agrees to receive intervention and be monitored by an initiator or mediator. $\mathrm{He} / \mathrm{she}$ should have been identified with a morbidity for which pharmaceutical therapy is being prescribed. The recipient can be the patient himself or the caretaker of the patient, and they are the focus of the model, so they represent a major part of it. The recipient has to provide factual information about themselves with regards to belief, financial status, family, etc. One of the major responsibilities of recipient is to assess the resources and capability of the initiator and moderator and hold them accountable for their responsibility.

One of the reasons we involved prescribers and specialists in our model is that our preliminary results showed that patients would prefer receiving drug information from a physician. The role of other health care professionals is well established in developed countries, unlike developing countries; hence, we suggested the role of a prescriber in our model to accommodate developing countries' health care system. It was also noted that a patient agreed to visit a drug specialist if requested and was allowed a time period of minimum 20 minutes for it. ${ }^{18}$

\section{Discussion}

Since 1950, numerous studies have been conducted toward understanding medication adherence, its factors, and ways for improvement. These studies had many differences in definitions, methodologies of conduction, assessment, duration, intervention, and interventionist. However, most of these studies had one basic similarity - being patient focused. All these studies were conducted to understand, assess, and intervene in the patient behavior. The most common definition used for medication adherence is presented by ISPOR, which defines it as "the extent to which patient acts in accordance with the prescribed interval and dose of a dosing regimen." Thus, substantiating medication adherence remains patient acentric.

Different variables relating to medication adherence were studied since 1975 , yet all failed to create a consistent link with medication adherence. ${ }^{19}$ This might have thus resulted in inconsistent outcomes shown in most meta-analyses or reviews. ${ }^{10}$ It has also been observed that interventions used in some situations would succeed, while in others they would not yield the anticipated outcome. Interventions may vary in terms of cost and utility in different health care systems or may be not applicable in routine medical practice. Most of these studies were disease- or medication-focused with a variable study period. Hence, we tried to change the paradigm by focusing on health care professionals.

All interventions developed to overcome factors causing barriers in medication adherence were based on theories. The most commonly used theory belongs to the psychological field, behavioral theories. With respect to our ideology, we were not able to find any theory or model that we could relate to. Hence, we initiated the development of a model which can be generalized for the health care professionals and patients in any given health care system irrespective of the intervention used.

During our discussion with different health care professionals and academicians, very little concerns were raised regarding the conceptual or graphical representation of our model. However, it was regularly compared with other practice-based models especially multidisciplinary model 
(MDM) and medication therapy management (MTM) model. Although, these three models may have similarities between them, we state that the core of all these models is different.

The MDM is a type of conceptual model in which health care professionals of different fields work together to achieve successful health outcomes for a patient. The MDM has been employed for improvement of medication adherence using pharmacists, nurses, and other allied health care professionals. ${ }^{3}$ Comparing our model with the MDM, it can be observed that the basic theme of both models is different. The model presented is based on a transdisciplinary approach (Figure 2) rather than a multidisciplinary approach (Figure 3). In the multidisciplinary approach, a team of different health care professionals work independently (assessment, plan development, implementation, and evaluation) in their fields, not sharing information with each other but being led by one health professional who is responsible for the outcome. However, in our model, responsibility and accountability is shared by the team, which does have a kind of managerial position to coordinate between all the individuals involved. Another difference is the involvement of the patient or his/her family, who play a vital role in our model, but is less likely in the MDM. One basic difference between the models is that MDM was developed to cater to the primary responsibilities of health care professionals, ie, clinical activities, whereas our model was developed to oversee secondary activities. Hence, it can be said that our model is different from the MDM.

The MTM model is a practice model extended from clinical pharmacy practice within a hospital setting to a community setting. Such medication management programs offered by a pharmacist have resulted in improvement in medication adherence and therapy continuation. ${ }^{20}$ Our model has a very close resemblance to MTM model wherein we

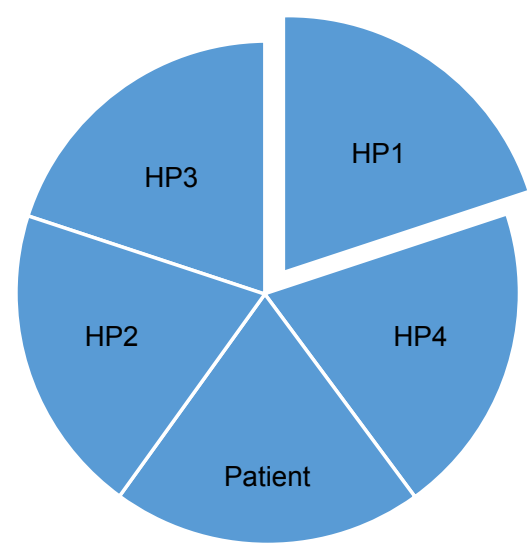

Figure 2 Graphical representation transdisciplinary model. Abbreviation: HP, health professional.

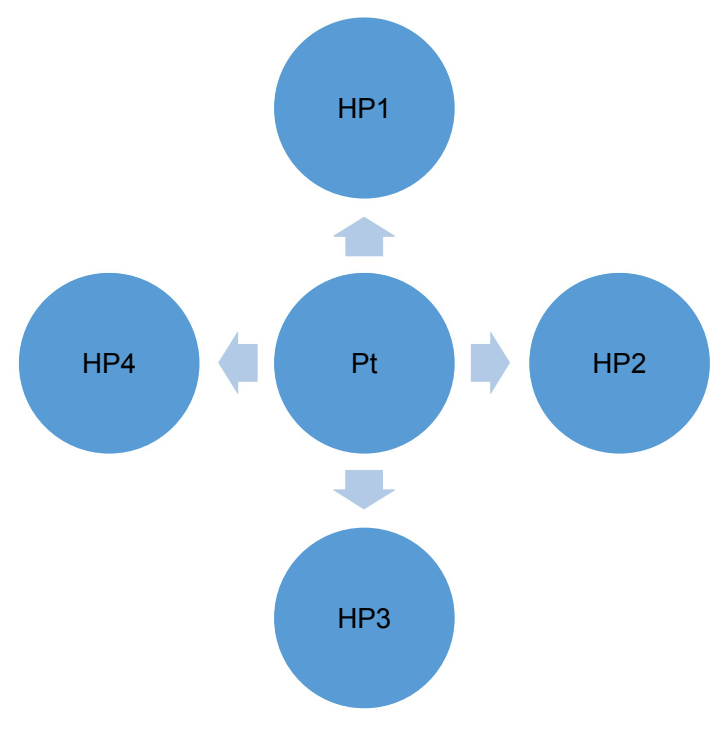

Figure 3 Graphical representation multidisciplinary model. Abbreviations: Pt, patient; HP, health professional.

have a pharmacist as a moderator. In the MTM model, the pharmacist is responsible for data collection, assessment, and decision-making for providing optimum pharmaceutical care, which also includes medication adherence. It also allows other qualified professionals to provide this service. The only major difference between our model and MTM model is the sharing of responsibilities. A meta-analysis has shown better result with various professionals being involved; also, our research has showed that the patient might be more prone to receiving information from a physician. Furthermore, we attempted to develop a generalized model which can be used in different health care systems, as pharmacists or certain specialized professionals may not be available in all systems due to human resource or financial constraint.

\section{Application of practice model of concordance}

Our conceptual model can be implemented by developing a procedural model comprising of the three main components ie, initiator, mediator, and recipient, and the environment mentioned. We have applied our practice model in a facility.

This outpatient facility of a hospital included pharmacy, laboratory and adult clinic of different specialties such as internal medicine, gynecology, orthopedics, psychiatry etc. All these services were interlinked with the electronic medical record (EMR). The normal procedure to see a physician in the facility was as follows: after registration, all patients undergo a triage in which current vital signs such as blood pressure, temperature, pulse rate, and respiratory rate were noted on the EMR along with other data such as height and weight; then 
patients were sent to the respective clinic. In the clinic, the physician would review the information entered by the triage nurse and enter orders (medication or laboratory test). These orders can be directly reviewed by the pharmacists or laboratory personnel for their respective activities.

Based on our conceptual model, we have developed a procedural model (Figure 4) which had a team as an initiator rather than an individual. The triage nurse acted as the "subinitiator," while the physician was the prescriber, and hence the "initiator." A clinical pharmacist acted as a "mediator," whereas the patient identified as having a medication adherence issue was the "recipient." When a patient was registered for any clinic, he/she underwent a common triage where the triage nurse ie, "subinitiator" collected data regarding patient adherence via a five-item medication assessment too ${ }^{21}$ along with other vitals scores, and the result was mentioned in the EMR. The initiator, ie, physician, while assessing the patient for any present issue or past issue also reviewed medication adherence assessment score if mediation was prescribed and diagnosed the patient as full adherent, partial adherent, or nonadherent (Figure 4). A fully adherent patient had level 1 or level 2 assessment, whereas level 3 was considered as partial, and levels 5 and 6 were considered as nonadherent. Partially adherent and nonadherent patients were the "recipients." Partial adherents were lightly counseled by the physician and directed to the clinic nurse for follow-up. In this nurse follow-up, the nurse called the patient after 2 or 3 days and determined adherence score for a physician to review. If a patient at any time was diagnosed as nonadherent, the physician sent a request with the help of clinic nurse for a "mediator" consultation which, in such a case, was the clinical pharmacist. The clinical pharmacist assessed the patient with respect to nonadherence issues and provided tailored interventions to the patient along with mandatory text message reminder about medication administration time. This was a continuous process for all new and old patients in which their medication adherence disorder was reviewed and accounted regularly. One of the major factors that assisted in the development of this procedural model and its implementation was the integrated EMR among nurse, physician, and pharmacist. The results of this project will be published in future.

This particular application of our model is one among many. The model concept can be used in different ways with respect to human, financial, and other resources.

The aim of the model was to develop a mechanism that allows health care professionals to assess, intervene, and monitor medication adherence disorder, as suggested by the World Health Organization. To our knowledge, such a practice model has not been developed and implemented to make medication adherence part of clinical practice involving assessment, diagnosis, and intervention.

\section{Ethical approval}

The Ethical Review Committee of Ziauddin University has reviewed this project in the meeting held on May 9, 2012 and gave approval to conduct this study (Reference number 0190212MAPHAR). All survey participants provided verbal

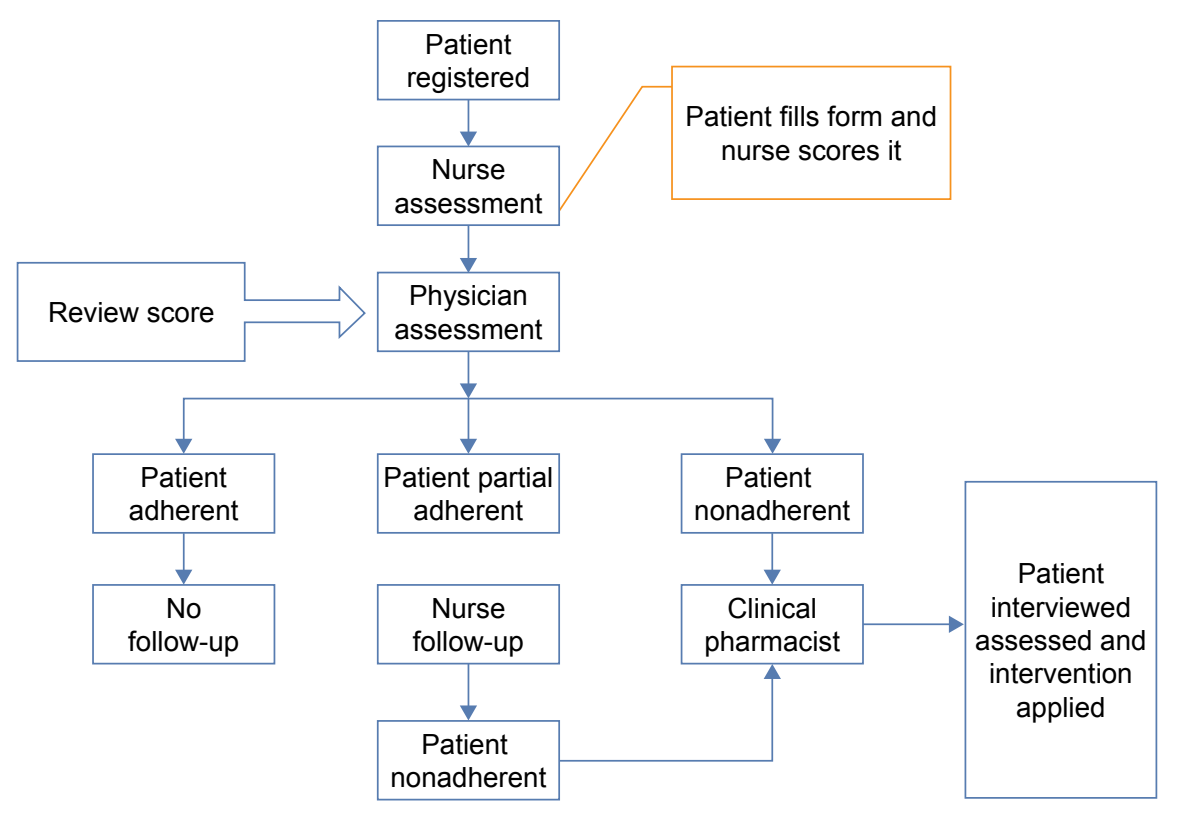

Figure 4 Process flow of medication adherence assessment and follow-up. 
consent after being informed of the purpose of the survey, as approved by the Ethical Review Committee of Ziauddin University.

\section{Acknowledgments}

The authors thank Professor Doctor Nathaniel Rickles (Associate Professor of Pharmacy Practice); Doctor Anum Misbah (Clinical Psychologist), Doctor Kamran Hameed, and the faculty members of Ziauddin College of Pharmacy for providing guidance in the development of the model. The authors also thank Professor Tran Viet-Thi and Doctor Stéphanie Sidorkiewicz for allowing the use of their medication adherence tool for our experiment.

\section{Author contributions}

Muhammad Amir developed and conceptualized the model presented in this paper. The paper was written by Muhammad Amir and was reviewed by Zeeshan Feroz and Anwer Ejaz Beg. All authors contributed toward data analysis, drafting and critically revising the paper, gave final approval of the version to be published, and agree to be accountable for all aspects of the work.

\section{Disclosure}

The authors report no conflicts of interest in this work.

\section{References}

1. Rickles NM, Svarstad BL. Relationships between multiple self-reported nonadherence measures and pharmacy records. Res Social Adm Pharm. 2007;3(4):363-377.

2. Osterberg L, Blaschke T. Adherence to medication. $N$ Engl J Med. 2005;353(5):487-497.

3. Costa E, Giardini A, Savin M, et al. Interventional tools to improve medication adherence: review of literature. Patient Prefer Adherence. 2015;9:1303-1314.

4. Shrank WH. Helping our patients to adhere to chronic medications: a new arrow for the quiver. J Gen Intern Med. 2011;26(12):1394-1395.

5. Rickles NM, Brown TA, McGivney MS, Snyder ME, White KA. Adherence: a review of education, research, practice, and policy in the United States. Pharm Pract. 2010;8(1):1-17.

6. Clyne W, Mshelia C, Hall S, et al. Management of patient adherence to medications: protocol for an online survey of doctors, pharmacists and nurses in Europe. BMJ Open. 2011;1(1):e000355.
7. Gwadry-Sridhar FH, Manias E, Lal L, et al. Impact of interventions on medication adherence and blood pressure control in patients with essential hypertension: a systematic review by the ISPOR medication adherence and persistence special interest group. Value Health. 2013;16(5):863-871.

8. Maes S. Health Psychology, back to the future. Eur Health Psychol. 2005;2:4-5.

9. Haynes RB, Ackloo E, Sahota N, McDonald HP, Yao X. Interventions for enhancing medication adherence. Cochrane Database Syst Rev. 2008;(2):CD000011.

10. McDonald HP, Garg AX, Haynes RB. Interventions to enhance patient adherence to medication prescriptions: scientific review. JAMA. 2002;288(22):2868-2879.

11. Møldrup C, Stein J, Søndergaard B. "Patients don't lie"; a view on adherence in asthma. Pharm World Sci PWS. 2010;32(6):795-798.

12. Fraser S. Concordance, compliance, preference or adherence. Patient Prefer Adherence. 2010;4:95-96.

13. Tamene E. Theorizing conceptual framework. Asian J Educ Res. 2016;4(2):50-56.

14. Jabareen Y. Building a conceptual framework: Philosophy, definitions, and procedure. Int J Qual Methods. 2009;8(4):49-62.

15. Khan KS, Kunz R, Kleijnen J, Antes G. Five steps to conducting a systematic review. J R Soc Med. 2003;96(3):118-121.

16. International Society for Pharmacoeconomics and Outcomes Research (ISPOR). Patient's Needs For Medication Adherence Are Still Unclear [press release]. Lawrenceville, NJ: International Society for Pharmacoeconomics and Outcomes Research (ISPOR); 2013 [March 17]. Available from: http://press.ispor.org/index.php/patients-needs-formedication-adherence-are-still-unclear/. Accessed September 24, 2018.

17. Weeks G, George J, Maclure K, Stewart D. Prescribing roles for health professionals other than doctors [review]. Cochrane. Available from: https://www.cochrane.org/CD011227/EPOC_prescribing-roles-healthprofessionals-other-doctors. Accessed September 13, 2018.

18. Amir M, Ata SA, Anum M. Disparity Between Health Care Professionals and Low Socioeconomic Patients with Respect to Factors Associated to Medication Non-Adherence in Karachi. ISPOR Connections. 2013;19(1):9-10.

19. Fenerty SD, West C, Davis SA, Kaplan SG, Feldman SR. The effect of reminder systems on patients' adherence to treatment. Patient Preference Adherence. 2012;6:127-135.

20. van Boven JF, Stuurman-Bieze AG, Hiddink EG, Postma MJ, Vegter S. Medication monitoring and optimization: a targeted pharmacist program for effective and cost-effective improvement of chronic therapy adherence. J Manag Care Spec Pharm. 2014;20(8):786-792.

21. Sidorkiewicz S, Tran VT, Cousyn C, Perrodeau E, Ravaud P. Development and validation of an instrument to assess treatment adherence for each individual drug taken by a patient. BMJ Open. 2016;6(5): e010510.
Patient Preference and Adherence

\section{Publish your work in this journal}

Patient Preference and Adherence is an international, peer-reviewed, open access journal that focuses on the growing importance of patient preference and adherence throughout the therapeutic continuum. Patient satisfaction, acceptability, quality of life, compliance, persistence and their role in developing new therapeutic modalities and compounds to optimize

\section{Dovepress}

clinical outcomes for existing disease states are major areas of interest for the journal. This journal has been accepted for indexing on PubMed Central. The manuscript management system is completely online and includes a very quick and fair peer-review system, which is all easy to use. Visit http://www. dovepress.com/testimonials.php to read real quotes from published authors. 